\title{
Variations
}

Variations

Revue internationale de théorie critique

$23 \mid 2020$

Pour une gauche érotique

\section{Corps à corps. Livre-phallus et oeil vaginal}

\section{Lucia Sagradini}

\section{(2) OpenEdition}

Journals

Édition électronique

URL : http://journals.openedition.org/variations/1796

DOI : 10.4000/variations. 1796

ISSN : 1968-3960

Éditeur

Les amis de Variations

\section{Référence électronique}

Lucia Sagradini, « Corps à corps. Livre-phallus et oeil vaginal », Variations [En ligne], 23 | 2020, mis en ligne le 01 septembre 2020, consulté le 07 septembre 2020. URL : http://journals.openedition.org/ variations/1796; DOI : https://doi.org/10.4000/variations.1796

Ce document a été généré automatiquement le 7 septembre 2020

Les ami•e•s de Variations 


\title{
Corps à corps. Livre-phallus et oeil vaginal
}

\author{
Lucia Sagradini
}

Dans ma pratique de chercheuse, il m'arrive de connaître la désorganisation interne provoquée par la pensée de l'autre. La dernière fois, c'était à la lecture du Mal d'archive de Derrida. Au sens plein du terme, j'ai lu ce livre et j'ai fait l'expérience d'un accident, d'un imprévisible. Car, lire est la possibilité de connaître le surgissement de l'imprévu. Le danger de la lecture est d'en subir le contrecoup, de connaître le court-circuit. L'auteur-e, sans toujours le savoir, peut éreinter son lecteur, sa lectrice. Car, la lecture est toujours potentiellement dangereuse, les autodafés de livres ont toujours, dans leur barbarie, montré ce pouvoir mutatif du livre pour celui ou celle qui lit.

2 Lisant les passages sur la circoncision, celle de Freud ou la sienne, j'ai sentie en boomerang, que je lisais un livre-Phallus, et tout d'un coup, mon oeil devenait vaginal. Je sentais un mâle d'archive et je me ressentais profondément lectrice. Le livre faisait de mon oeil un oeil marqué par l'absence de phallus. Les interrogations légitimes de Derrida concernant l'inscription de l'homme par le décallotage du pénis me laissaient sans voix, profondément trouée.

Des questions absurdes et implicites accompagnaient la lecture : une femme peut-elle avoir une archive ? Une femme est-elle en capacité de penser ou d'archiver, alors qu'elle n'a pas «l'engin», la techné nécessaire, pour prétendre occuper une place si fortement marquée par la présence de l'attribut masculin par excellence ? La réaction était totalement irrationnelle, passionnelle : je m'interrogeais sur la possibilité de penser un phallus féminin. Fallait-il penser que les femmes partaient parfois à la recherche de leur phallus perdu dans une prolongation improbable de la théorie freudienne de la castration? Lire devenait lire avec mon sexe. Une chatte parmi les chattes ! Etait-ce un rabaissement ? Un échec de la neutralité, un endroit sans recherche que ma réaction? Je décidais de repenser plus tard l'impact imprévisible de ce livre tout en reconnaissant le choc qu'il provoquait : une désorganisation interne profonde, un trouble de la pensée face à la pensée de l'autre dans un endroit assez imprévisible. 

de ce petit texte que je vais m'y employer. Je pense que ce «mâle d'archive» me contraint en faisant de moi un "oeil vaginal» à penser la condition féminine de chercheuse qui me traverse.

La pensée de Derrida est comme une injonction à penser le creux : qu'est-ce que je peux penser comme le «mon corps » d'une chercheuse ? Impossible pour moi d'imaginer, à la suite de Cixious, le clitoris comme un petit pénis, un clitoris qui viendrait confirmer que la femme est un homme comme les autres. L'idée sous-jacente de cette théorie est en réalité une manière de laisser toujours aux mains de la virilité la puissance. Si la femme a un pénis (son clitoris) alors tout est sauf, les pages virilistes qui peuplent la littérature et la pensée et qui associent les vertus de la créativité, du courage et de la force (par exemple) à la masculinité tiennent encore. Nombreuses sont les solutions théoriques mais aussi pratiques, où les femmes construisent une réponse à la domination par l'appropriation des attributs virils. Mais la trame de ces solutions est que la vertu reste alors, ce qu'en pensaient déjà les Romains, une chose virile. Si la possibilité d'un devenir homme ne doit pas s'exclure dans les parcours personnels, néanmoins, et, en pensant aux travaux de Marie-Hélène Bourcier, l'enjeu est d'imaginer une autre possibilité pour nous, toutes et tous. Il nous faut donc aller plus loin. Il nous faut donc aller ailleurs. Sur une autre plage.

Pour le coup, que signifie virilité en latin? Virilité signifie le fait d'affronter. Faire face. Il s'agit de la manifestation d'une puissance de l'être. Quand je relis Virginia Woolf, je pense très simplement que la puissance n'a rien à voir avec un sexe, ni avec une histoire de pénis, avec ou sans prépuce. La puissance est une affaire d'énergie. Dans la situation de la recherche, il s'agit de parvenir à lier une énergie avec une chaîne de significations encore inconnues. Réussir à donner forme à des intuitions qui se matérialisent en puisant dans cette énergie et en se transformant au contact de l'objet d'étude.

détonante, impossible à nier ou à retenir. Le corps. Le corps d'une chercheuse est venue se manifester pour se rendre présent à la pensée. Comme si, il se faisait tête pour propager une idée simple : lire n'est pas neutre et tout l'être de celui ou de celle qui lit se trouve engagé. Que, peut-être aussi, les idées ne viennent pas toutes de l'esprit, mais que, parfois, les idées surgissent d'autres organes, d'autres parts de l'être que le siège de sa raison. Peut-être, Derrida prenant dans un corps-à-corps livresque cette question de son propre corps, des traces qu'il porte, permet, dans le contrecoup, au corps de la chercheuse de devenir abstrait, espace à penser pour elle-même, et plus nié en tant que surface à réfléchir. Le rappel du « mon corps » de la chercheuse dans son incarnation a ceci de paradoxal de le faire entrer dans le champ (la visibilité) de la réflexion par la rencontre étrange avec Le mal d'archive. Tout d'un coup, Derrida faisait que le «mon corps » s'imposait. Il était là, entre les lignes de Derrida. Phénomène incongru. Télescopage.

8 Pour le coup, les pratiques artistiques semblent avoir un large temps d'avance : alors que la sociologie a beaucoup travaillé la question de l'in-corporation, c'est-à-dire, de s'employer à montrer comment le corps est le lieu où se concentrent les formes de la domination; les pratiques artistiques proposent une autre voie, celle de penser les formes de "l'ex-corporation », les manières dont le sujet exprime son autonomie par son corps. La lutte contre l'aliénation est donc une lutte pour la libération des corps, où 
l'être peut trouver le chemin pour cesser d'être un objet et devient un sujet au sens plein. La recherche, touchant au corps comme espace, nécessite de prendre conscience des formes de domination qui le traversent, mais aussi des formes de libération qui l'occupent. L'écriture peut alors devenir être un espace de jeu pour construire ces représentations qui font de nous des êtres libres. Avec ou sans phallus.

INDEX

Mots-clés : Théorie critique, féminisme, Jacques Derrida, livre-pahllus, oeil vaginal

\section{AUTEUR}

LUCIA SAGRADINI

Lucia Sagradini est professeure d'histoire de l'art à l'Ecole supérieure d'art des Pyrénées ESAD 